\title{
IMF Control and Convection in the Jovian Magnetosphere
}

\author{
A. NISHIDA ${ }^{1}$ and K. MAEZAWA ${ }^{2}$ \\ ${ }^{1}$ Institute of Space and Astronautical Science, Komaba, Meguro, Tokyo, Japan \\ ${ }^{2}$ Department of Physics, Nagoya University, Nagoya, Japan
}

(Received July 22, 1985; Revised March 26, 1986)

\begin{abstract}
The dependence of the Jovian non-Io-related decametric emission on solar wind parameters demonstrates that the energy of the solar wind is imparted to the Jovian magnetosphere. This paper considers if, and then how, the steady-state reconnection model can be used for the understanding of this energy transfer process. Referring to the observationally obtained values of key parameters as much as possible, we investigate if the steady state model as commonly applied for the earth's magnetosphere can be adopted as a reasonable approximation for the Jovian magnetosphere as well. We find that rapid rotation of the planet does not present a serious obstacle to a steady progress of reconnection since the motion of the field lines is governed by the magnetosheath plasma and the slipping occurs at the ionospheric ends. Next we discuss the convection generated by reconnection for northward and southward polarities of the interplanetary magnetic field, and address the field line motion in the tail and the current system generated in the ionosphere. Influence of the centrifugal force on the motion of field lines in the inner region of the magnetosphere is also discussed.
\end{abstract}

\section{Introduction}

The planetary magnetosphere is a dynamical system where plasma is convected, energetic particles are produced, and waves are generated. The energy to support these activities can come from two possible sources. One is the kinetic energy of the solar wind which flows toward the magnetosphere, and the other is the energy of the planetary rotation imparted to the magnetospheric plasma. The former is by far the dominant source in the case of the Earth's magnetosphere, and the open model of the magnetosphere has been developed in which some of the geomagnetic field lines get reconnected with the field lines of the interplanetary magnetic field (IMF). This model has successfully explained a variety of correlations which have been found between the state of IMF and the activities of the magnetosphere. Recent developments have been reviewed by COWLEY (1982) and NISHIDA (1983).

In the case of the Jovian magnetosphere, both energy sources seem to be important. The activities of the Jovian magnetosphere have been monitored by the intensity of decametric and hectometric radio emissions which serve as means for the remote sensing. According to observations these emissions can be divided into two categories, namely into those which are correlated with the orbital phase of the 
satellite Io and those which are not. The Io-related component has been attributed to the energy extracted from the planetary rotation by Io (accompanied by its plasma population) whose orbital speed is slower than the corotational velocity (e.g., NeUBAUER, 1980; GURNETT and GoERTZ, 1981). The intensity of the non-Io related component, on the other hand, has been found to depend on solar wind parameters directly or indirectly, namely on dynamic pressure (TERASAWA et al., 1984), density (DESCH and BARROW, 1984), and the geomagnetic $K p$ index (OYA and MORIOKA, 1981). This dependence demonstrates that the Jovian magnetosphere acquires energy also from the solar wind.

The purpose of this paper is to study if the reconnection mechanism which is thought to operate in the Earth's magnetosphere can be considered as a viable means for the energy transfer from the solar wind to the Jovian magnetosphere. Although a direct comparison between the IMF condition and the decametric emission is difficult to perform because of the high variability of IMF, from the experience of studying the response of the Earth's magnetosphere to solar wind conditions it seems possible that the dependence on the IMF condition is coupled with, or hidden behind, the detected dependence on other solar wind parameters. The effect of reconnection on the Jovian magnetosphere was discussed earlier by BRICE and IOANNIDIS (1970) who superposed the potentials of the interplanetary and the corotational electric fields in the equatorial plane. The present paper begins by inquiring if such steady-state like treatment can be justified, and then proceeds to develop a three-dimensional picture of the reconnected field lines and the associated convection.

\section{Basic Considerations}

Once connection is established between field lines of the planetary magnetic field and the interplanetary magnetic field, momentum and energy are transmitted from the solar wind into the magnetosphere to set up convective motion in the magnetospheric plasma. In the steady state the profile of the magnetospheric convection resulting from this process can be derived conveniently by projecting the potential of the electric field along the connected field lines, since the electric field $\boldsymbol{E}$ and the flow velocity $\boldsymbol{U}$ are related by the frozen-in relation $\boldsymbol{E}=-\boldsymbol{U} \times \boldsymbol{B}$ where $\boldsymbol{B}$ is the magnetic induction. The underlying assumption is that the electric potential drop along magnetic field lines is sufficiently small and / or localized, so that its neglect does not introduce serious error in the derivation of the overall convection profile.

The method of the electric-potential projection has been used widely in investigations of the convection profile in the Earth's magnetosphere (e.g., NiSHIDA, 1971; STERN, 1973; COWLEY, 1981). For interpreting the observed dependence of the polar-cap convection profile on the direction of IMF in the upstream solar wind, the topology of the connected field lines has been drawn and the electric potential has been projected along them. Since the method has proved to be quite successful when applied to the Earth's magnetosphere, we would like to adopt it for the investigation of the IMF control and convection in the Jovian magnetosphere. Before doing so, however, we have to confirm if the steady state picture of reconnection with IMF can 
be regarded as a valid approximation in the Jovian magnetosphere.

When compared to the Earth's magnetosphere, the Jovian magnetosphere has several distinguishing characteristics. Because of the gigantic dimension it takes several hours for the solar wind to flow past merely the dayside portion of the Jovian magnetosphere. Furthermore the time scale of the transpolar motion of the reconnected field lines is as long as a week, reflecting the small ratio of the IMF strength to the field strength at the Jovian polar ionosphere (KENNEL and CORONITI, 1979). When combined with the Jovian rotational period of 10 hours these time scales may be taken to suggest the IMF field lines would be twisted and distorted while they are connected to the polar ionosphere as illustrated in Fig. 1. If the twisting is very severe, an explosive process would be needed for the detwisting, and the convection would not proceed steadily.

Let us consider the behaviour of a magnetic field line after being reconnectd at the reconnection line located on the dayside magnetopause. As soon as the field line is reconnected, the electric potential of the magnetosheath side starts to propagate toward the planetary ionosphere with the Alfven speed through the cusp region. The Alfven speed in the cusp region cannot yet be derived from observed parameters but a lower limit can be obtained by referring to low-latitude, dayside observations by Pioneers and Voyagers outside the plasmadisc. Beyond the distance of $20 R_{J}$ the magnetic induction is expressible roughly by $B=8 \times 10^{1}(20 / \mathrm{L})^{2} \mathrm{nT}$ (SMITH et al., 1974 ) and the plasma density is roughly $n=1 \times 10^{5}(20 / \mathrm{L})^{4} / \mathrm{m}^{3}$ (GURNETT et al., 1981).

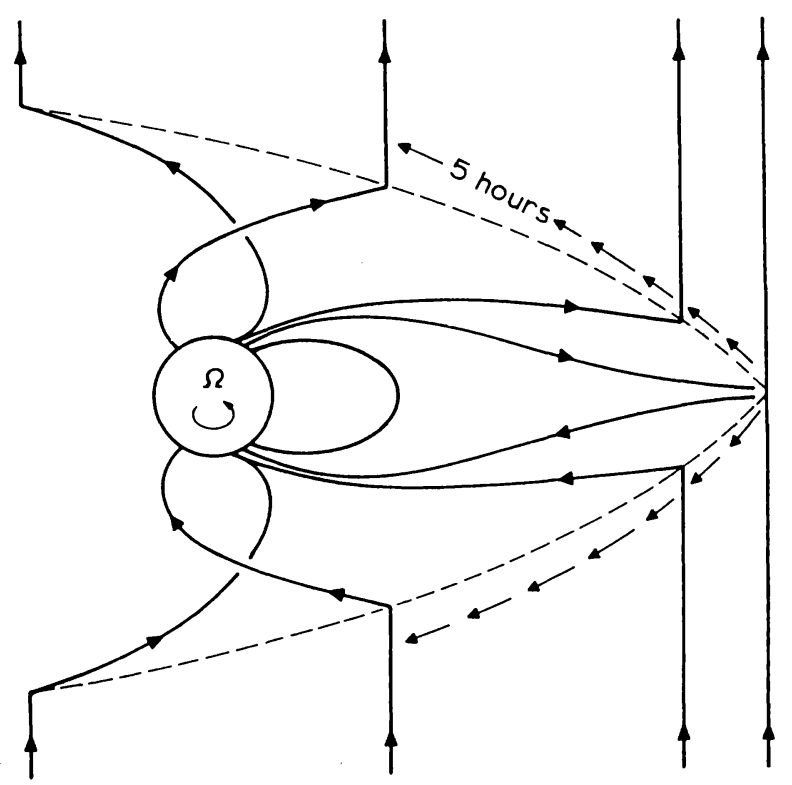

Fig. 1. Distortion of open field lines of the Jovian magnetosphere envisaged earlier by KENNEL and Coroniti (1975). Solid lines are magnetic field lines and dashed lines illustrate the magnetopause. 
This means that the Alfven speed $V_{\mathrm{A}}$ in the cusp region (where the density is presumably lower than in low latitudes) would be higher than $5.5 \times 10^{6} \mathrm{~m} / \mathrm{s}$, and if the reconnection line is located at $60 \mathrm{R}_{\mathrm{J}}$ the transit time of the electric potential from there to $20 R_{J}$ is less than $500 \mathrm{~s}$. The additional time required for propagation from $20 R_{J}$ to the ionosphere is expected to be much smaller than this because of a sharp increase in the magnetic field strength toward the planet.

When the electric field $\boldsymbol{E}$ arrives at the ionosphere the partial reflection occurs. The electric field $\boldsymbol{E}$ entering the ionosphere is related to the geometrically scaled value $\boldsymbol{E}$ of the electric field incident from the solar wind by

$$
E=E_{\mathrm{C}}+T\left(E_{\mathrm{S}}-E_{\mathrm{C}}\right)
$$

where $E_{\mathrm{C}}$ is the electric field associated with the corotational motion. In (1) the electric field is expressed in the inertia frame. To derive the above, we write the same equation in the corotating frame where the electric field is primed:

$$
E^{\prime}=T E_{\mathrm{S}}^{\prime}
$$

and use the relations $E=E^{\prime}+E_{\mathrm{C}}$ and $E_{\mathrm{S}}=E_{\mathrm{S}}^{\prime}+E_{\mathrm{C}}$. The transmission coefficient $T$ at the ionosphere is given by

$$
T=\frac{2 \Sigma_{\mathrm{A}}}{\Sigma_{\mathrm{A}}+\Sigma}
$$

where $\Sigma$ is the height-integrated ionospheric Pedersen conductivity and $\Sigma_{\mathrm{A}}=\left(\mu_{0} V_{\mathrm{A}}\right)^{-1}$ is the Alfven conductance. The ratio $\Sigma_{\mathrm{A}} / \Sigma$ is not a large number; using $B=8 \times 10^{-4} \mathrm{~T}$, $n=1 \times 10^{10} / \mathrm{m}^{3}$ and $\Sigma=0.1 \mathrm{mho}$, we obtain $\Sigma_{\mathrm{A}} / \Sigma=5 \times 10^{-2}$ and $T=0.1$. Thus only one tenth of the incident electric field enters the ionosphere while the rest is reflected toward the magnetosheath. Adopting the usual convection of interpreting the plasma drift velocity $\boldsymbol{U}=(\boldsymbol{E} \times \boldsymbol{B}) / \boldsymbol{B}^{2}$ as the velocity of magnetic field lines, we can say that field lines cannot be dragged readily by the solar wind because of the line tying at their ionospheric ends. However, the interaction does not end here.

The electric field reflected to the magnetosheath works to decelerate the solar wind, but the bulk flow of the solar wind has an enormous momentum that can easily overcome this deceleration. The cancellation of the reflected wave in the magnetosheath produces a second set of $\boldsymbol{E}_{\mathrm{S}}$ which also propagates toward the ionosphere, and multiple propagations occur until the field lines move completely with the magnetosheath plasma. (An analogous problem was solved by WATANABE and NISHIDA (1982) who studied the temporal development of the departure from the corotation that is created by an inward motion of the Jovian plasma disc.) The time scale of this process is given roughly by $2 T^{-1}$ times the propagation time, and this can be estimated to be less than $1 \times 10^{4} \mathrm{~s}$ in the present case. Since the time scale is smaller than the period of the Jovian rotation, we shall combine the multiple incident signals and assume that a single signal establishes the coupling between the ionosphere and the 
magnetosheath. The dominance of the magnetosheath plasma means that the transmission coefficient $T$ thus redefined is almost 1 and hence $E \sim E_{\text {s. }}$.

The reason why the corotational electric field is relatively unimportant can be seen as follows. The polarization charges accumulated by the dynamo current $\Sigma U_{\mathrm{C}} B$ that flows in the ionosphere produce the electric field $E_{\mathrm{R}}$. Here we refer to the inertial frame and denote the corotational velocity by $\boldsymbol{U}_{\mathrm{C}}$. If field lines are not reconnected and furthermore no torque operates within the magnetosphere, we have $\boldsymbol{E}_{\mathrm{R}}+\boldsymbol{U} \times \boldsymbol{B}=0$, that is, the electric field vanishes in the corotating frame, so that no more polarization proceeds in the ionosphere. In this case the entire magnetosphere would be set to corotate with the planet in association with the spreading of the $\boldsymbol{E}_{\mathrm{R}}$ field throughout the magnetosphere along the magnetic field lines. However, when the reconnection has taken place the angular momentum of the rotational motion is lost to the magnetosheath plasma along the connected field lines. This means that polarization charges are continually lost from the ionosphere, and $\boldsymbol{E}_{\mathrm{R}}$ is not allowed to build up to the level of $\boldsymbol{E}_{\mathrm{C}} \equiv-\boldsymbol{U}_{\mathrm{C}} \times \boldsymbol{B}$.

Figure 2 schematically illustrates a volume bounded by four reconnected field lines. A box at the bottom represents the ionospheric end of the volume where $s_{\mathrm{a}}$ and $s_{\mathrm{b}}$ denote the size of the box in latitudinal and longitudinal directions, respectively. The dynamo electric field $U_{\mathrm{C}} B$ is in the latitudinal direction and is to be partially compensated by the polarization electric field $E_{\mathrm{R}}$. Another box illustrated in the upper portion of the volume is where the field lines are kinked as the torque from the ionosphere is being applied to the solar wind plasma. This kink in field lines is propagating upward with the Alfven speed. All the parameters in the kink region are primed.

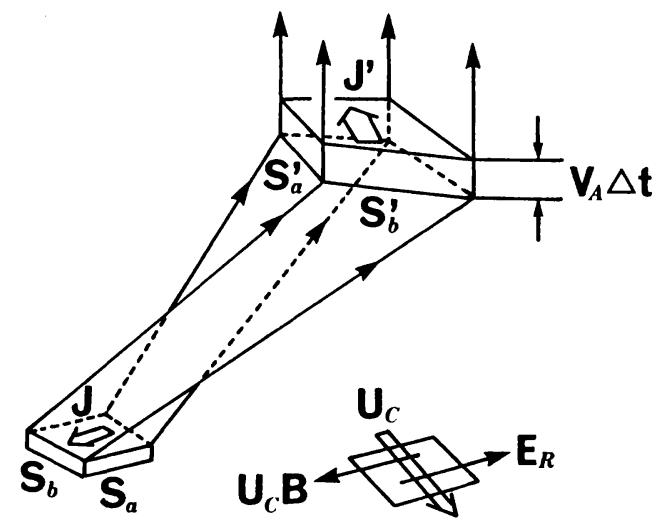

Fig. 2. A diagram to illustrate the coupling between the ionosphere and the magnetosheath. The polarization produced by the dynamo electric field $\boldsymbol{U}_{\mathrm{C}} \times \boldsymbol{B}$ in the ionosphere (lower box) is transmitted to the magnetosheath along the connected field lines and is discharged as it modifies the motion of the sheath plasma (upper box). Because of this discharging effect the polarization electric field $\boldsymbol{E}_{\mathrm{R}}$ cannot become large enough to cancel the dynamo field. 
The electric current flows across the magnetic field both in the ionosphere and in the kink region. The continuity of the current requires that these are in balance:

$$
J^{\prime} s_{\mathrm{b}}^{\prime}=\Sigma\left(E_{\mathrm{R}}+U_{\mathrm{C}} B\right) s_{\mathrm{b}}
$$

and since field lines are equipotentials except inside the kink region,

$$
E_{\mathrm{R}}^{\prime} S_{\mathrm{a}}^{\prime}=E_{\mathrm{R}} S_{\mathrm{a}} .
$$

Inside the kink region of thickness $V_{\mathrm{A}}^{\prime} \Delta t$ the velocity is changed in time $\Delta t$ from 0 to $E_{\mathrm{R}}^{\prime} / B^{\prime}$ by the Lorentz force $J^{\prime} B^{\prime}$. The equation of motion is, therefore,

$$
\rho^{\prime} V_{\mathrm{A}}^{\prime} \frac{E_{\mathrm{R}}^{\prime}}{B^{\prime}}=-J^{\prime} B^{\prime}
$$

Combining these, we obtain

$$
E_{\mathrm{R}}=\frac{-U_{\mathrm{C}} B}{\frac{1}{\mu_{0} V_{\mathrm{A}}^{\prime}} \frac{1}{\Sigma} \frac{s_{\mathrm{a}}}{s_{\mathrm{a}}^{\prime}} \frac{s_{\mathrm{b}}^{\prime}}{s_{\mathrm{b}}}+1} .
$$

Typical values of plasma density and magnetic field in the magnetosheath outside the Jovian magnetosphere are $1 \times 10^{6} / \mathrm{m}^{3}$ and $3 \mathrm{nT}$, respectively. Using these and $\Sigma=0.1$ mho we obtain $1.2 \times 10^{2}$ as the ratio $\left(\mu_{0} V_{A}^{\prime} \Sigma\right)^{-1}$ of conductances. Hence, unless the ratio $R=\left(s_{\mathrm{a}} / s_{\mathrm{a}}^{\prime}\right) /\left(s_{\mathrm{b}} / s_{\mathrm{b}}^{\prime}\right)$ of scaling factors is much less than $10^{-2},\left|E_{\mathrm{R}}\right|$ is substantially less than $\left|U_{\mathrm{C}} B\right|$, namely the polarization electric field is not established. Since $\boldsymbol{E}_{\mathrm{R}}+\boldsymbol{U}_{\mathrm{C}} \times \boldsymbol{B}$ is the condition for the corotation, this means that magnetic field lines in the magnetosphere do not corotate with the planet.

Rather than estimating $R$ directly for the geometry of Fig. 2, we evaluate the scaling factor for an ionospheric area whose boundaries are aligned to the solar magnetospheric $x$ and $y$ directions. If $10 \%$ of the IMF flux flowing toward the 200 $\mathrm{R}_{\mathrm{J}}$-wide Jovian magnetosphere is reconnected and projected to the $0.5 \mathrm{R}_{\mathrm{J}}$-wide polar cap, the scaling factor in the dawn-dusk (namely, $y$ ) direction is

$$
\frac{s_{y}^{\prime}}{s_{y}}=40
$$

The scaling factor $s_{x}^{\prime} / s_{x}$ in the noon-midnight (namely, $x$ ) direction follows from this and the ratio $B^{\prime} / B=4 \times 10^{-6}$ of the field strength;

$$
\frac{s_{x}^{\prime}}{s_{x}}=\frac{s_{y}}{s_{y}^{\prime}} \frac{B}{B^{\prime}}=6 \times 10^{3}
$$

Thus the scaling factor is very much greater in the noon-midnight direction than in 
the dawn-dusk direction. (If we wish to make these scaling factors comparable maintaining the assumption of the $10 \%$ reconnection rate which has been widely accepted in the Earth's case, it is necessary to assume that the flow of the reconnected flux in the ionosphere is confined inside a $4 \times 10^{-2} \mathrm{R}_{\mathrm{J}}$ channel.)

In the dawn or dusk meridian the latitudinal direction coincides with the solar magnetospheric $y$-direction and the longitudinal direction with the $x$-direction. Hence $R=\left(s_{y} / s_{y}^{\prime}\right) /\left(s_{x} / s_{x}^{\prime}\right)$ and this is very much greater than $10^{-2}$, so that $\left|E_{\mathrm{R}}\right| \ll\left|U_{\mathrm{C}} B\right|$. In the noon-midnight meridian the correspondences are between latitudinal and $x$-directions and between longitudinal and $y$-directions. Here the ratio $R=\left(s_{x} / s_{x}^{\prime}\right) /\left(s_{y} / s_{y}^{\prime}\right)$ is $7 \times 10^{-3}$, and this suggests that in the noon meridian $\boldsymbol{E}_{\mathrm{R}}+\boldsymbol{U}_{\mathrm{C}} \times \boldsymbol{B}=0$ approximately holds and the corotational motion can be imposed on reconnected field lines. However, the region where $\left(\mu_{0} V_{\mathrm{A}}^{\prime} \Sigma\right)^{-1} R \leq 1$ is apparently limited to the proximity of the noon meridian. Moreover, although in the present formulation $E_{\mathrm{R}}$ is assumed to be transmitted only along the reconnected field lines, in reality the motion enforced from the ionosphere spreads into the bulk of the solar wind by creating compression or rarefaction waves. In other words, non-reconnected field lines should also be taken along. Momentum of the rotational motion supplied to the reconnected field lines is diluted by this effect. Therefore, the rotational motion is not likely to give decisive influence on the dynamics of the reconnected field lines at any local time.

HINES (1960) has shown earlier that when the upper end of magnetic field lines is clamped the rotational motion of the ionospheric plasma impressed by the atmospheric motion falls off exponentially with a scale height of $\left(\eta / \sigma B^{2}\right)^{1 / 2}$, where $\eta$ is coefficient of viscosity and $\sigma$ is conductivity. This scale height is estimated to be about $1 \mathrm{~km}$ by substituting $B=8 \times 10^{-4} T$ and assuming $\eta \sim 10^{-5}$ and $\sigma \sim 10^{-5}$ in the MKS unit.

Using $s_{y}^{\prime}$ and $B^{\prime}$ already assumed and also $200 \cdot \mathrm{km} / \mathrm{s}$ as the flow speed in the magnetosheath, we can estimate that a potential difference of $0.8 \mathrm{MV}$ is introduced by reconnection with IMF. We also have

$$
\frac{v^{\prime}}{v}=\frac{B}{B^{\prime}} \frac{s_{y}}{s_{y}^{\prime}}=6 \times 10^{3}
$$

Thus $v^{\prime}=200 \mathrm{~km} / \mathrm{s}$ in the magnetosheath is translated to $v=30 \mathrm{~m} / \mathrm{s}$ in the ionosphere, so that it takes $1 \times 10^{6} \mathrm{~s}$ or 12 days to traverse the $0.5 \mathrm{R}_{\mathrm{J}}$ wide polar cap. This is two orders of magnitude longer than the multiple propagation time, estimated earlier in this section, of the electric field between the magnetosheath and the ionosphere. This further justifies the assumption that transients associated with this multiple propagation can be ignored.

From these considerations it seems reasonable to contruct a steady state model where the interplanetary electric potential is projected along reconnected field lines down to the ionospheric level and the motion of the reconnectd field lines is not significantly influenced by the planetary rotation. The underlying assumption is that the potential drop along field lines is not large enough to substantially modify the global electric field distribution. 


\section{Effect of the Northward IMF}

Since the Jovian magnetic dipole is directed almost opposite to that of the Earth, the effect of the northward IMF on the Jovian magnetosphere is essentially similar to the effect of the southward IMF on the Earth's magnetosphere. Figure 3 illustrates the expected convection streamlines when the Jovian magnetosphere is interacting the IMF with the northward polarity. The IMF $y$ polarity is assumed to be positive; the picture for the negative $y$ polarity can be obtained easily by taking a mirror image with respect to the equatorial plane. The polar cap is lightly shaded in Fig. 3 and subsequent figures.

Figure 3(A) is the view from the front. In the northern polar cap the field lines reconnected at the reconnection line (RL) move tailward and duskward, and they

(A) DAYSIDE MAGNETOPAUSE

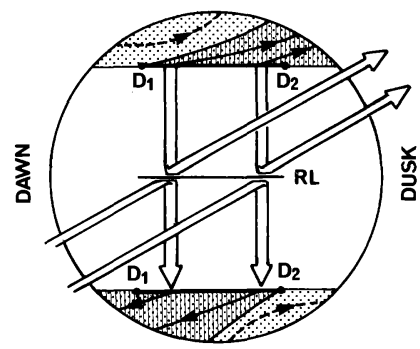

(B) TAIL CROSS SECTION

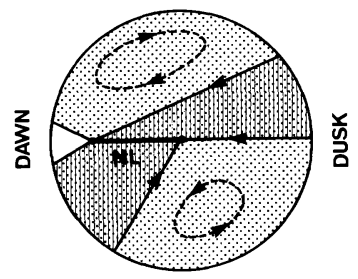

(C) NORTHERN IONOSPHERE

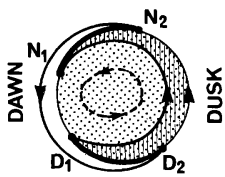

NOON

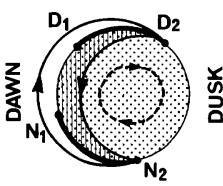

(D) SOUTHERN IONOSPHERE

RECONNECTION WITH NORTHWARD IMF

Fig. 3. Convection streamlines in the Jovian magnetosphere under the northward IMF condition. (A) is the view of the frontside magnetosphere and (B) is the section of the tail as viewed through the front side. (C) and (D) show streamlines in the northern and southern ionospheres, respectively. The types of field lines are represented by shadings as defined at the lower right. The nature of the open but not-reconnected field lines is discussed in the text. 
enter the tail from the duskside tail boundary as illustrated in Fig. 3(B). In the southern hemisphere the motion is tailward and dawnward. The convection paths of these field lines are marked by heavy shadings in these and later figures. Unless the reconnection line encircles the entire dayside magnetopause the reconnected field lines would not cover the entire polar cap. The rest of the polar cap would consist of those field lines which were opened earlier by reconnection when the IMF had a different polarity, or by other viscous-like processes or the centrifugal force which can also stretch the field lines. The plasma density on these field lines would be low as the connection with the IMF occurs, if at all, far downstream in the tail. Thus the plasma density in the tail lobe is expected to have a spatial non-uniformity which depends on the $y$ polarity of IMF. Such a structure has been detected in the Earth's tail lobe (HARDY et al., 1979; GosLING, 1984).

The rotational motion originating from the planet could be imposed in the region of these not-recently-reconnected field lines. Even if these field lines may be clamped to the solar wind at the other end, this would not inhibit the rotational motion if the solar wind is moving away with the super-Alfvenic speed and its influence cannot reach the ionosphere. Hence we have drawn streamlines of the rotational motion in the low density region.

The tail cross section of Fig. 3(B) includes the nightside reconnection line NL where the field lines approaching from the north and the south meet together and are reconnected again to form closed field lines. Since this reconnection line need not be a straight line, the figure represents a curved surface rather than a plane. The wedgeshaped unshaded region to the left of the reconnection line accommodates closed field lines which are stretched by the centrifugal force. The convection of these closed field lines will be discussed in later section.

Figures 3(C) and 3(D) show the motion of the ionospheric footpoints of field lines in the northern and southern polar regions. The streamlines enter the polar cap in the $D_{1} D_{2}$ sector which is the projection of the magnetopause reconnection line, and they exit the polar cap in the $N_{1} N_{2}$ sector which is the projection of the reconnection line in the tail. Under the given polarity of IMF, the streamlines of the reconnected field lines are on the dusk side of the northern polar cap and on the dawn side of the southern polar cap. Flows on these streamlines have the same direction as the corotation in the northern polar cap but are substantially slower. In the southern polar cap the flow direction is opposite to the corotational direction.

Outside the polar cap where the field lines are not clamped to the solar wind, the corotational motion is dominant. In the return flow region between $N_{1} N_{2}$ and $D_{1} D_{2}$ the flow speed would be slightly raised above the corotational speed in order to carry the return flux. The latitudinal extent of the return flow region is expected to be substantially narrower than that of the tailward flow in the polar cap, because the corotational speed is much higher than the estimated tailward flow speed in the polar cap. The return flow would not take place on the dusk side where the strong corotational flow cannot be overcome, although the flow would be slowed somewhat outside the duskside polar cap. The equatorial projection of these streamlines outside the polar cap can be recognized in the convection profile drawn by BRICE and 
IOANNIDIS (1970). The system of ionospheric Hall currents that correspond to these streamlines is illustrated in Fig. 4 on the assumption that the ionospheric conductivity is uniform. To obtain Fig. 4 from Figs. 3(C) and 3(D), we have subtracted outside the polar cap the corotational motion which does not contribute to the current and reversed the direction of the flow since electrons carry the Hall current. The current system is essentially the same as what has been obtained for the Earth's case.

\section{Effect of the Southward IMF}

The reconnection with the southward IMF would take place in the Jovian case on the surface of the magnetotail. The topology of the reconnectd field lines is illustrated in Fig. 5. When the IMF $y$ polarity is negative, the reconnected field lines in the northern polar cap move dawnward and those in the southern polar cap move duskward along the polar-cap boundary. Figure 6(A) illustrates the front view of this reconnection process. The reconnection lines $L_{1} L_{2}$ and $L_{3} L_{4}$ are inclined to the polar cap boundary and the recently-reconnected field lines occupy the wedge-shaped regions between the reconnection line and the polar cap boundary. Since reconnections in northern and southern polar caps can be thought to occur independently in this case the reconnection lines $L_{1} L_{2}$ and $L_{3} L_{4}$ need not be symmetrical. As long as reconnection occurs only in the polar caps, between the boundaries of the northern and the southern polar caps the magnetic field has no component normal to the magnetopause and hence the electric field has no tangential component. Points $L_{2}$ and $L_{3}$ therefore have the same electric potential.

Figure 6(B) shows the convection profile in the tail. After entering the tail the reconnected field lines flow toward the tail surface (not toward the neutral sheet) to get reconnected again. Since the present process does not involve transitions between open and closed field lines no other reconnection line exists inside the tail. Not all the polar cap field lines need to take part in this reconnection process, and the rest of the polar cap consists of not-recently-reconnected field lines. The convection of such field

\section{HALL CURRENTS (IMF $\left.B_{Z}>0, B_{Y}>0\right)$}

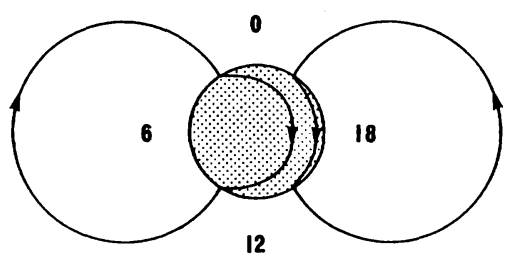

NORTHERN IONOSPHERE

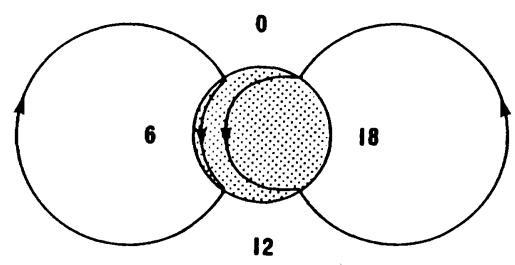

SOUTHERN IONOSPHERE

Fig. 4. The ionospheric Hall currents that would be produced by reconnection under the northward IMF condition. 


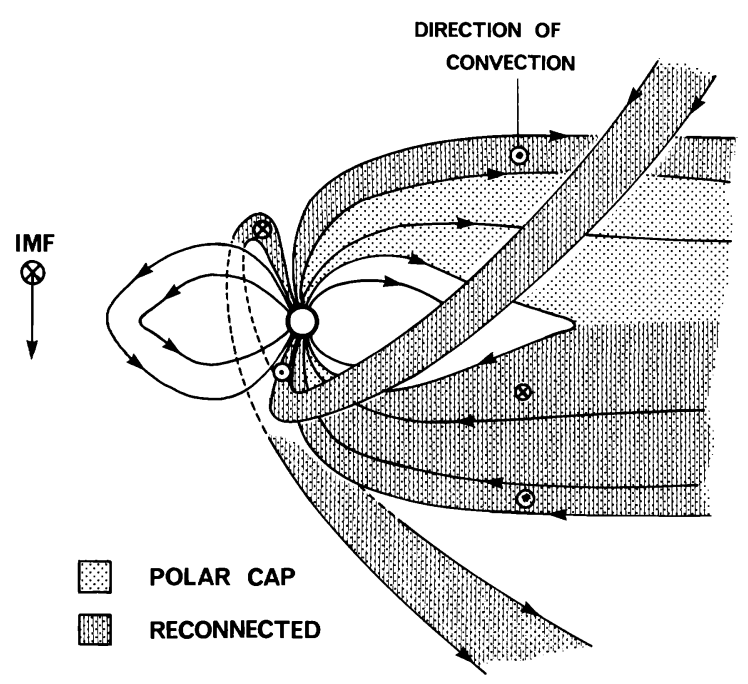

MAGNETOSPHERE UNDER SOUTHWARD IMF

Fig. 5. Topology of the reconnected field lines under the southward IMF condition.

lines is assumed to be dominated by the corotational motion for the reason mentioned in the previous section. Figures 6(C) and 6(D) show convection profiles in the ionosphere. Projections $L_{1} L_{2}$ and $L_{3} L_{4}$ of the reconnection lines are inside the polar cap rather than at its boundary, and the streamlines are confined to the polar cap. In Fig. $6(\mathrm{C})$ the return flow of the reconnected field lines spreads over the entire polar cap as it has the same sense as the corotational flow. If the conductivity is uniform, the Hall current system can be obtained essentially by reversing the direction of the streamlines in Figs. 6(C) and 6(D) in the heavily shaded region and expanding the current vortex slightly into the region of the not-recently-reconnectd field lines where the corotational motion has been modified.

Recently REIFF and BURCH (1985) discussed convection streamlines in the earth's magnetotail under the northward IMF polarity, which translates to the southward IMF polarity in the Jovian case. Comparison of their summary diagram (their Fig. 9) with our Fig. 5 shows basic similarities but also some differences. The differences are, (1) our model has not incorporated the viscous interaction with the solar wind, but (2) has explicitly identified the open but-not-recently-reconnected field lines and also incorporated the centrifugal stretching of closed field lines. Moreover, (3) our model does not involve the "reclosure cell" of closed field lines which are supposed by Reiff and Burch to be produced by reconnection at the tail neutral sheet even when the IMF polarity is northward (in the Earth's case), as we do not find a compelling reason for adopting this concept in the Jovian case. 


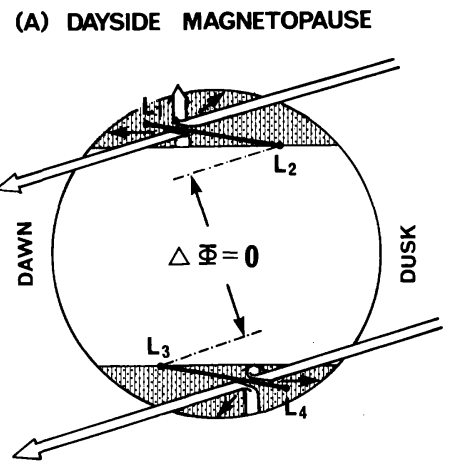

(B) TAIL CROSS SECTION

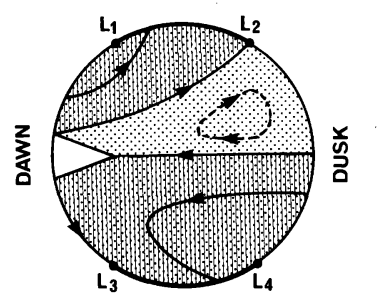

(C) NORTHERN

IONOSPHERE

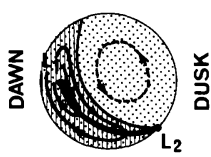

NOON

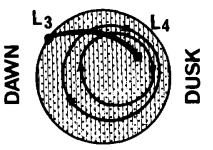

(D) SOUTHERN IONOSPHERE

\section{RECONNECTION WITH SOUTHWARD IMF}

Fig. 6. Similar to Fig. 3 but for the reconnection under the southward IMF condition.

\section{Convection of Closed Field Lines}

Since the potential difference of the corotational electric field (360 MV) is considerably greater than the potential difference introduced by reconnection with the northward IMF, the convection of the field lines which do not participate directly in the reconnection process is expected to be dominated by the corotation (BRICE and IOANNIDIS, 1970). These field lines are loaded in their equatorial section with dense plasma originating from the Io's plasma torus. The centrifugal force acting on this disc plasma would stretch the field lines while they travel the nightside (Fig. 7(A)). As the field lines approach the dawn side their length has to be shortened sharply in order to be accommodated within the dimension of the dayside magnetosphere. However, they would not be able to shrink smoothly due to the influence of the centrifugal force acting outward. The same problem exists also for those closed field lines which are produced by reconnection at the nightside reconnection line (NL of Fig. 3); Since these field lines are located above the centrifugally stretched field lines carried by corotation from the dayside they cannot shrink smoothly either. 

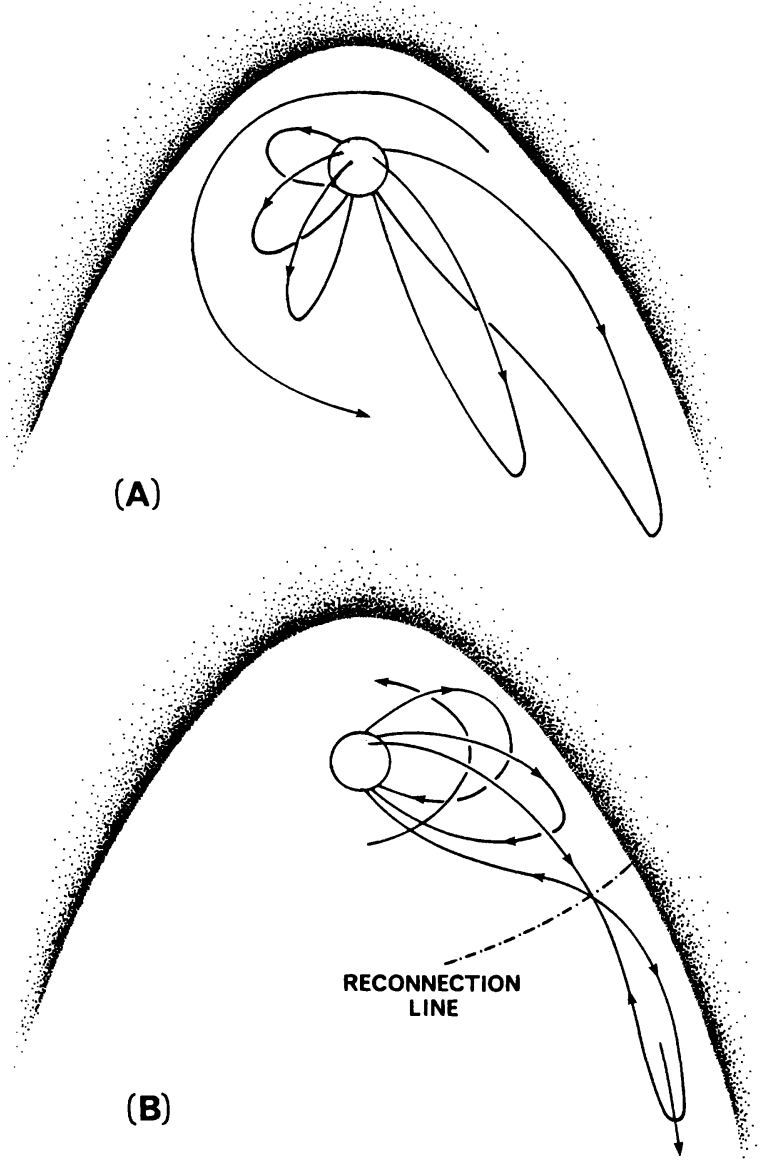

Fig. 7. Convective motion of the outer portion of the closed field lines. (A) Rotation of field lines from the dayside to the nightside, and (B) cutting of the tips of these field lines by reconnection.

It seems likely that reconnection occurs also on the stretched, closed field lines (Fig. 7(B)); the length of the field lines can be drastically reduced by separating the extended tip (CARBARY et al., 1976; VASYliUnAS, 1982). This scenario has been compared with field and particle observations of Voyager 1 and 2, and the expected signature, namely the outward flow of northward-oriented field lines, has been identified near the dawn side magnetopause (NISHIDA, 1983). The occurrence of this signature is highly time dependent, indicating that the reconnection occurs sporadically. In Figs. 3(B) and 6(B) presented earlier the wedge-shaped region inside the dawnside tail surface represents the escape route of the reconnected tip of the formerly closed field lines. 
Thus the closed field lines which return to be dayside can be divided into two categories, those which have experienced reconnection to reduce their length and those which have been kept closed all the time. The flux tubes of the first category are expected to contain less plasma as they lose particles each time their tip is separated by reconnection. To the first approximation therefore one would expect that there is a layer of the reduced plasma density in the outermost part of the closed field line region on the dayside.

The spatial distribution of plasma in the Jovian outer magnetosphere, however, is more complicated. Instead of a distinct separation of the low density outer layer from the high density inner body, a mixture has been observed inside the dayside magnetopause. Namely, although there is a low density region beyond the outer boundary of the plasma disc it contains numerous spiky density enhancements where the electron temperature is low as compared to where the density is not enhanced. It has been noted that the enhanced density and reduced temperature within the spikes are similar to those observed in the outermost region of the plasma disc (SCUDDER et al., 1981). A very similar structure has been detected also in the Saturnian magnetosphere.

A possible interpretation of this structure is that the flux tubes have been interchanged as suggested earlier by SCUDDER et al. (1981). The layering of the low density region above the high density region is potentially unstable when the centrifugal force exceeds the gravitational force, and localized regions of high plasma density could represent flux tubes of the outer plasma disc which have been detached by a centrifugally driven interchange instability (GOERTZ, 1983). The ballooning instability could also contribute in mixing the two plasma regimes.

\section{Discussion}

Pioneer and Voyager observations have yielded two pieces of information which seems to be consistent with the connection of the Jovian magnetic field with IMF. The first of these is the intensity of the energetic particle flux observed in the dawn sector outside the equatorial high flux region. This intensity is as low as the flux detected in the interplanetary space. If the sharp latitudinal decline in the flux intensity is related to the pitch angle anisotropy, an extremely strong anisotropy should have been observed in the equatorial region. Since such anisotropy has not been detected, it seems more likely that field lines of higher latitudes are connected to IMF and energetic particles have freely escaped to interplanetary space, as suggested by GOERTZ et al. (1976).

Another relevant information has been obtained at distances of hundreds of $R_{J}$ from the nominal position of the dawnside magnetopause. KURTH et al. (1981) has detected density depressions down to the magnetosphere-like level and/or the continuum radiation of the magnetospheric origin in the region which is normally filled by the solar wind. As we have estimated earlier one end of the IMF field line remains connected to the Jovian magnetosphere for an interval of about $10^{6} \mathrm{~s}$ after the reconnection. During this interval the main body of the field line located outside the 
bow shock travels a distance of $3000 \mathrm{R}_{\mathrm{J}}$. The observed features can be interpreted as encounters with these connected field lines where the solar wind plasma density has been rarefied and the continuum radiation from Jupiter has been guided. (If the density on the reconnectd field lines is so rarefied, the contrast in density between the reconnected region and the not-recently-reconnected open field line region of Figs. 3(B) and 6(B) would not be very pronounced). Although we cannot deny alternative interpretations suggested by Kurth et al., the reconnection model seems to offer a straightforward explanation which is particularly attractive for interpreting events detected at thousands of $R_{J}$ from Jupiter. A similar feature may be present outside the Earth's magnetosphere, but it would not be as pronounced as the connection time is much less, namely about $2 \times 10^{4} \mathrm{~s}$.

Studying the dynamics of the magnetosphere by a steady-state model can only be an approximation because the IMF condition is highly variable. However, the IMF structures that can influence the macroscopic structure of the magnetosphere should have time scales longer than the equilibration time between the interacting solar wind and magnetosphere elements, and it is the effect of such structures with which we have been concerned in this paper. Variations in the IMF polarity during the estimated connection time of weeks would result in a complex configuration of open field lines as orientations of their constituents differ widely reflecting the history of the IMF polarity during the interval of weeks. The reconnection can still be expected to proceed smoothly as long as the reconnected field lines are transported anti-sunward one after another, without acting as an obstacle to the convection of field lines that follow. If the reconnection proceeds sporadically in time and/or locally in space in some limited part of the magnetopause, the reconnected flux tubes would be isolated and look like flux transfer events identified for the Earth's magnetosphere by RUSSELL and ELPHIC (1979). The dynamics of such isolated flux tubes would be controlled even more tightly by the bull: flow of the solar wind as compared to when most of the IMF field lines are reconnected, because unreconnected field lines which constitute the majority would collaborate to carry the reconnectd field lines with them.

Our model has "not-recently-reconnected" field lines in the polar cap. These field lines may have been made open by such mechanisms as the viscous-like drag by the solar wind and the centrifugal force acting on the interior plasma. Anti-planetward escape of the field line loop (i.e., plasmoid) created at the nightside neutral line would also contribute to force these field lines in the high latitude tail to be kept open. It may also be possible that some of the field lines reconnected to the northward IMF sometimes do not have a chance to be reconnected again on the nightside because of changes in the convection pattern resulting from the IMF changes.

As noted earlier the existence of not-recently-reconnected field lines in the earth's tail has been inferred from the dawn/dusk and north/south asymmetry in the density of magnetosheath-like plasma detected in the lobe. It is also consistent with the recently reported relationship between the $y$ component of IMF and the $y$ component of the lobe field observed in the distant tail by ISEE-3. When the IMF $y$ polarity is positive, its influence on the tail $B_{y}$ can be recognized in the north-dawn and 
south-dusk quadrants of the lobe but not in the north-dusk nor south-dawn quadrants. Similar asymmetry is noted also when IMF $y$ polarity is negative (TSURUTANI et al., 1984). By translating Figs. 3 and 6 to the Earth's magnetosphere we can readily confirm that the quadrants where the dependence on the IMF $B_{y}$ cannot be recognized correspond to the domain of the not-recently-reconnected field lines.

\section{REFERENCES}

Brice, N. M. and G. A. IoANnidis, The magnetospheres of Jupiter and Earth, Icarus, 13, 173-183, 1970. Carbary, J. F., T. W. Hill, and A. J. Dessler, Planetary spin period acceleration of particles in the Jovian magnetosphere, J. Geophys. Res., 81, 5189-5195, 1976.

COWLEY, S. W. H., Magnetospheric asymmetries associated with the $y$-component of the IMF, Planet. Space Sci., 29, 79-96, 1981.

COWLEY, S. W. H., The cause of convection in the earth's magnetosphere: A review of developments during the IMS, Rev. Geophys. Space Phys., 20, 531-565, 1982.

Desch, M. D. and C. H. BARRow, Direct evidence for solar wind control of Jupiter's hectometer wavelength radio emission, J. Geophys. Res., 89, 6819-6823, 1984.

GoertZ, C. K., Detached plasma in Saturn's front side magnetosphere, Geophys. Res. Lett., 10, 455, 1983.

Goertz, C. K., D. E. Jones, B. A. Randall, E. J. Smith, and M. F. Thomsen, Evidence for open field lines in Jupiter's magnetosphere, J. Geophys. Res., 81, 3393-3398, 1976.

GosLING, J. T., ISEE-3 plasma measurements in the lobes of the distant geomagnetic tail: inferences concerning reconnection at the dayside magnetopause, in Magnetic Reconnection in Space and Laboratory Plasmas, edited by E. W. Hones, pp. 157-158, Am. Geophys. Union, 1984.

GurnetT, D. A. and C. K. GoertZ, Multiple Alfven wave reflections excited by Io: Origin of the Jovian decametric arcs, J. Geophys. Res., 86, 717-722, 1981.

Gurnett, D. A., F. L. Scarf, W. S. Kurth, R. R. Shaw, and R. L. Poynter, Determination of Jupiter's electron density profile from plasma wave observations, J. Geophys. Res., 86, 8199-8212, 1981.

Hardy, D. A., H. K. Hills, and J. W. Freeman, Occurrence of the lobe plasma at lunar distance, $J$. Geophys. Res., 84, 72-78, 1979.

Hines, C. O., On the rotation of the polar ionospheric region, J. Geophys. Res., 65, 141-144, 1960.

KENNEL, C. F. and F. V. CoRONITI, Is Jupiter's magnetosphere like a pulsar's or earth's?, Space Sci. Rev., 17, 857-883, 1975.

KenNel, C. F. and F. V. Coronitı, Jupiter's magnetosphere and radiation belts, in Solar System Plasma Physics, II, edited by C. F. Kennel, L. J. Lanzerotti, and E. N. Parker, pp. 105-181, North-Holland Pub. Co., 1979.

Kurth, W. S., D. A. Gurnett, F. L. Scarf, R. L. Poynter, and J. D. Sullivan, Voyager observations of Jupiter's distant magnetotail, J. Geophys. Res., 86, 8402-8412, 1981.

Neubauer, F. M., Nonlinear standing Alfven wave current system at Io: Theory, J. Geophys. Res., 85, 1171-1178, 1980.

NishiDA, A., Interplanetary origin of electric fields in the magnetosphere, Cosmic Electrodyn., 2, 350-389, 1971.

NishidA, A., Reconnection in the Jovian magnetosphere, Geophys. Res. Lett., 10, 451-454, 1983.

NishidA, A., IMF control of the earth's magnetosphere, Space Sci. Rev., 34, 185-200, 1983.

OYA, H. and A. MORIOKA, Relations between turbulent regions of interplanetary magnetic field and Jovian decametric radio wave emissions from the main source, Planet. Space Sci., 29, 783-791, 1981.

REIFF, P. H. and J. L. BURCH, IMF $B_{y}$ dependent plasma flow and Birkeland currents in the dayside magnetosphere, 2, Global model for northward and southward IMF, J. Geophys. Res., 90, 1595-1609, 1985.

RuSSELL, C. T. and R. C. ELPHIC, ISEE observations of flux transfer events at the dayside magnetopause, Geophys. Res. Lett., 6, 33-36, 1979. 
Scudder, J. D., E. C. SitTler, Jr., and H. S. Bridge, A survey of the plasma electron environment of Jupiter: A view from Voyager, J. Geophys. Res., 86, 8157-8179, 1981.

Smith, E. J., L. Davis, Jr., D. E. Jones, P. J. Coleman, Jr., D. S. Colburn, P. Dyal, C. P. Sonett, and A. M. A. Frandsen, The planetary magnetic field and magnetosphere of Jupiter: Pioneer $10, J$. Geophys. Res., 79, 3501-3513, 1974.

Stern, D. P., A study of the electric field in an open magnetospheric model, J. Geophys. Res., 78, 7292-7305, 1973.

Terasawa, T., K. Maezawa, and S. Machida, Solar wind effect on Jupiter's non-Io related radio emission, Nature, 273, 131-132, 1978.

Tsurutani, B. T., D. E. Jones, R. P. Lepping, E. J. Smith, and D. G. Sibeck, The relationship between the IMF $B_{y}$ and the distant tail (150-238 $\mathrm{R}_{\mathrm{e}}$ ) lobe and plasma sheet $B_{y}$ fields, Geophys. Res. Lett., 11, 1082-1085, 1984.

Vasyliunas, V. M., Plasma distribution and flow, in Physics of the Jovian Magnetosphere, edited by A. J. Dessler, pp. 395-453, Cambridge University Press, 1982.

Watanabe, Y. and A. Nishida, Field line distortion and Joule heating by the corotation enforcement current in the Jovian magneto-ionosphere, J. Geophys. Res., 87, 8111-8117, 1982. 